\title{
The in-vivo Mode of Action of Quinoline Antimalarial Drugs Unveiled by X-ray Microscopy
}

Sergey Kapishnikov ${ }^{1}$, Trine Staals $\varnothing^{2}$, Yang Yang ${ }^{2}$, Jiwoong Lee ${ }^{2}$, Ana J Pérez-Berná ${ }^{3}$, Eva Pereiro ${ }^{3}$, Stephan Werner ${ }^{4}$, Peter Guttmann ${ }^{4}$, Leslie Leiserowitz ${ }^{5}$ and Jens Als-Nielsen ${ }^{2}$

${ }^{1}$ University of Copenhagen / Chemical Research Support, Weizmann Institute of Science, Rehovot, HaMerkaz, Israel, ${ }^{2}$ University of Copenhagen, Copenhagen, Hovedstaden, Denmark, ${ }^{3}$ ALBA Synchrotron Light Source, Barcelona, Catalonia, Spain, ${ }^{4}$ Helmholtz-Zentrum Berlin, Berlin, Berlin, Germany, ${ }^{5}$ Weizmann Institute of Science, Rehovot, HaMerkaz, Israel

Emerging resistance of malaria-causing parasites Plasmodium to current drug treatments highlights the need for identifying efficient targets to improve antimalarial therapies. Malaria symptoms appear when Plasmodium parasites are released into the blood stream where they invade the red blood cells. Within a red blood cell the parasite consumes hemoglobin. Hemoglobin digestion liberates large quantities of heme, which is toxic to the parasite. The parasite detoxifies free heme by its crystallization into inert hemozoin that remains in the parasite and offers a docking surface for continuously supplied heme.

Our recent study, based on soft X-ray cryo-tomography and X-ray fluorescence microscopy ${ }^{1}$, indicates that hemoglobin digestion and hemozoin crystallization is an assembly line process, where the rates of heme liberation and heme crystallization obviously must match. Both processes occur in a single parasitic organelle called the digestive vacuole wherein large quantities of hemoglobin have been found. ${ }^{2}$ Therefore, if hemozoin crystal growth is severely retarded, this would result in accumulation of the toxic heme in the parasite leading to poisoning of the latter. One way to inhibit hemozoin crystal growth is by capping its surface with an appropriate molecule thereby reducing the number of available docking sites. This is a promising drug target.

In order to understand how biogenic hemozoin crystal growth can be efficiently inhibited, its structure as well as the mechanism of biogenic hemozoin nucleation, and its immediate intracellular environment need to be investigated. The structure of synthetic hemozoin has been solved by Pagola et al. ${ }^{3}$. The crystal structure of the biogenic counterpart has been refined by Straas $\varnothing$ et al ${ }^{4,5}$ and Marom et al $^{6}$ using powder X-ray diffraction and DFT-based computations. A study where a combination of X-ray diffraction, soft $\mathrm{X}$-ray tomography and electron microscopy was employed, had demonstrated that the majority of hemozoin crystals nucleate at the inner leaflet of the digestive membrane, probably due to a partial stereochemical complementarity between the membrane head groups and the carboxylic acid groups at the $\{100\}$ faces of the centrosymmetric crystal. ${ }^{7-9}$ Furthermore, multiple soft X-ray cryo-tomography maps of the parasites indicate that the biogenic hemozoin crystals grow in an aqueous medium ${ }^{1,2}$. Consequently, water-soluble molecules capable of docking on the hemozoin crystal faces need to be used in order to inhibit the crystallization process.

Over the past twenty years the question of quinoline drug action in the malaria parasite has been narrowed down to whether quinoline drugs interfere with the nucleation and growth of hemozoin crystals. It was suggested, based on computational docking studies of chloroquine ${ }^{10}$ and ferroquine ${ }^{11}$, that quinoline- 
family drugs dock onto the large $\{100\}$ side faces as well as the relatively small $\{001\}$ and $\{011\}$ terminating faces of the needle-like crystals of hemozoin.

To investigate how quinoline family drugs act in-vivo we undertook a correlative soft X-ray cryotomography and cryo X-ray fluorescence nanoprobe study ${ }^{12}$ of a well-established quinoline family drug within rapidly frozen Plasmodium-infected red blood cells (iRBCs) not subject to chemical fixation, staining, drying or sectioning. We chose chloroquine as a model drug. However, due to natural abundance of chlorine, chloroquine was replaced by its bromine $(\mathrm{Br})$ analog bromoquine to enable detection of the drug by X-ray fluorescence of $\mathrm{Br}$ atoms. We found that bromoquine accumulates within the parasitic digestive vacuole at concentrations thousand-fold higher than in the culture medium. Hemozoin crystals, sharp-edged optically dense objects, visible in soft X-ray tomograms (Fig. 1A), contain high density of iron (Fe), which translates into a high X-ray Fe fluorescence signal (Fig. 1B). In bromoquine-treated samples, X-ray Br fluorescence maps of hemozoin-rich areas demonstrate correspondingly high levels of Br signal replicating the location and shape of hemozoin crystals (Fig. 1C). The quantity of bromoquine capping hemozoin crystals was calculated from X-ray Br fluorescence maps. The number of bromoquine docking sites on intracellular hemozoin crystals was calculated from their surface area obtained from soft $\mathrm{X}$-ray tomograms. This translated into bromoquine surface coverage of $10 \pm 5 \%$ of the available docking sites. Crucially, bromoquine was found capping the same surface fraction in hemozoin crystals floating in the culture medium outside iRBCs (Fig. 2A). Here we used X-ray fluorescence maps to calculate the surface area of these stand-alone crystals. This unique finding indicates strong affinity of bromoquine to the surface of hemozoin crystals, despite the low drug concentration in the extracellular medium. In bromoquine-treated iRBCs, elevated $\mathrm{Br}$ signal was also found at the membrane of the digestive vacuole (Fig. 2B). Such distribution of bromoquine suggests that the remaining drug, in the lumen of the digestive vacuole, complexes with the heme deprived from crystallization. This complex is driven towards the digestive vacuole membrane increasing the chances of membrane puncture and heme spillage into the parasitic interior resulting in self-poisoning of the malaria parasite. It is noteworthy that additional $\mathrm{Br}$ signal was observed lining parasitic membranes other than the digestive vacuole. This signal arises from $\mathrm{Br}$ present in bovine serum in the culture medium. Importantly, this background does not overlap with the bromoquine signal since in bromoquine-free samples no $\mathrm{Br}$ was observed at the digestive vacuole membrane or hemozoin crystals (Fig. 2C). This study demonstrates how mapping three-dimensional cellular structure by soft X-ray cryo-tomography combined with elemental distribution mapping by cryo $\mathrm{X}$-ray fluorescence within the same fully hydrated intact cells can address important questions such as the in-vivo mode of antimalarial drug action. ${ }^{12}$
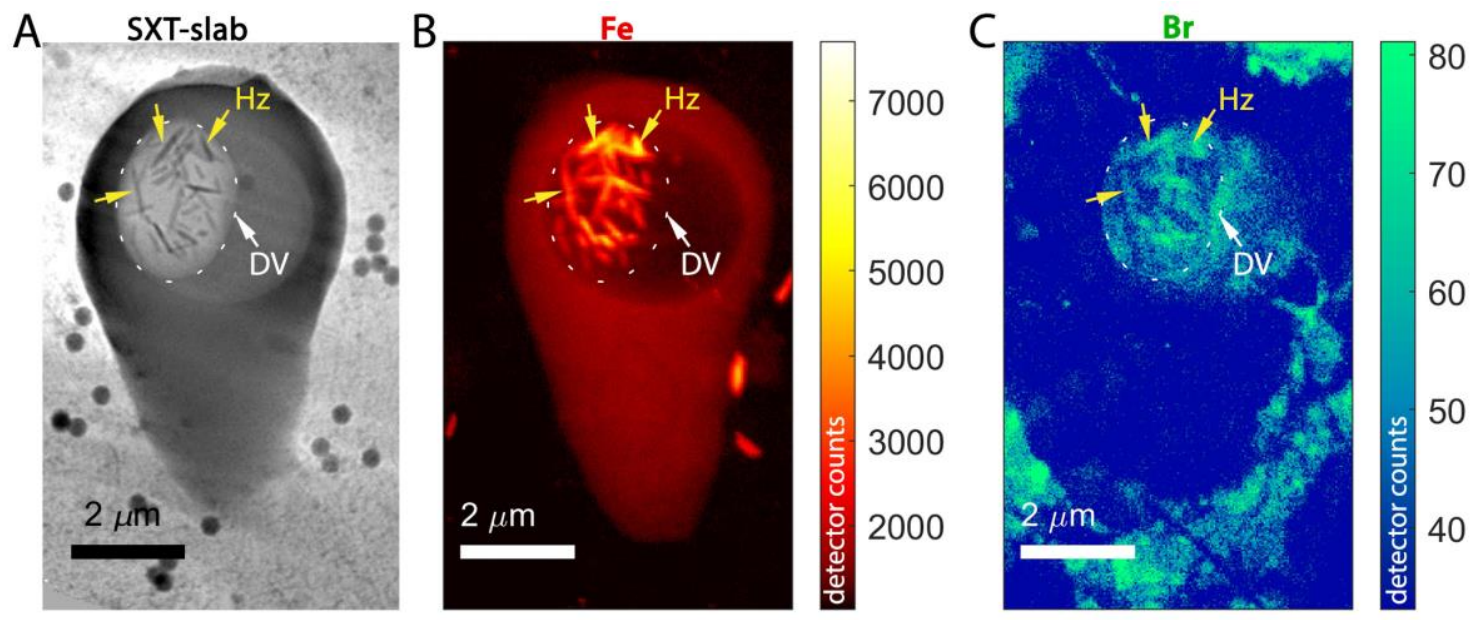
Figure 1. (A) $2 \mu \mathrm{m}$ thick soft X-ray tomogram (SXT) virtual slab of a Plasmodium-infected red blood cell (iRBC). Digestive vacuole (DV) is outlined here and in other panels with a dashed line. For guidance, a few of many hemozoin crystals $(\mathrm{Hz})$ located in the DV are pointed at by yellow arrows. (B) Iron (Fe) X-ray fluorescence map of the cell shown in panels A and C. (C) Bromine (Br) X-ray fluorescence map of the cell shown in panels $\mathrm{A}$ and $\mathrm{B}$.
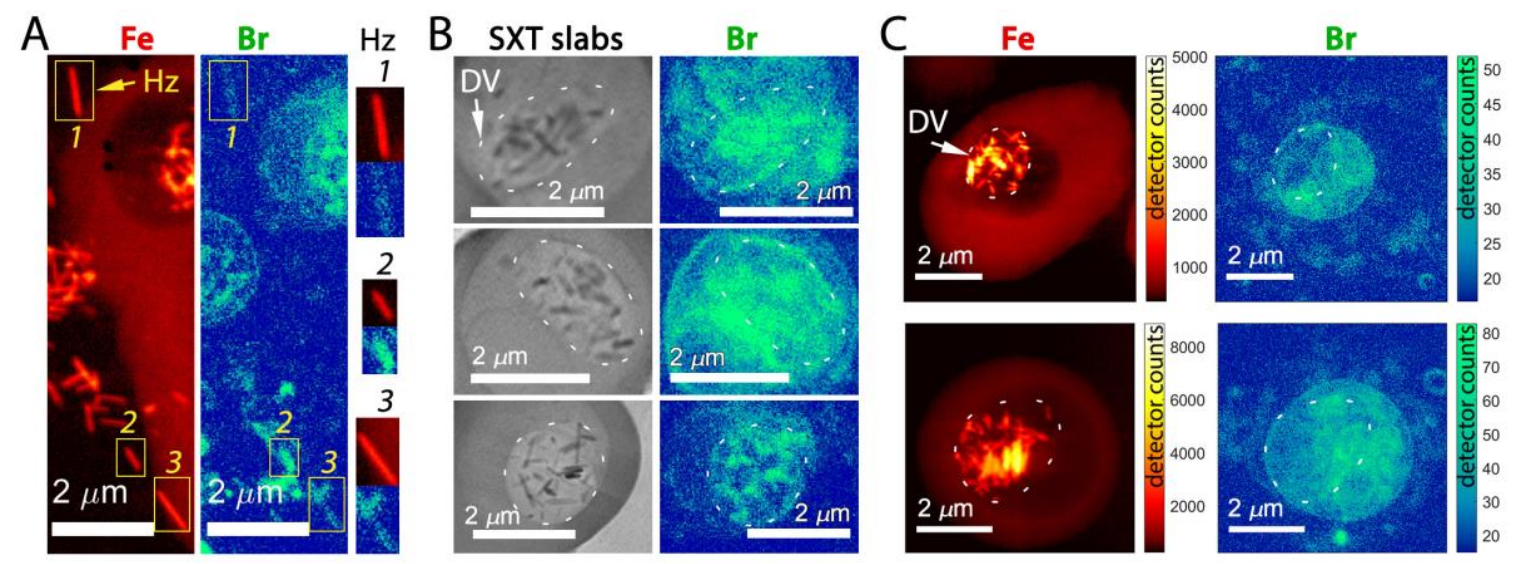

Figure 2. (A) Fe and $\mathrm{Br}$ maps of stand-alone Hz crystals. The Hz crystals 1-3 are shown magnified at the right side of the panel. (B) $\sim 1 \mu \mathrm{m}$ thick SXT virtual slabs and Br X-ray fluorescence maps of parasitic DVs in bromoquine-treated samples $(\mathrm{C}) \mathrm{Fe}$ and $\mathrm{Br} \mathrm{X}$-ray fluorescence maps of parasitic DVs in bromoquine-free samples.

\section{References}

1 Kapishnikov, S. et al. Biochemistry of malaria parasite infected red blood cells by X-ray microscopy. Sci. Rep. 7, 802 (2017)

2 Kapishnikov, S. et al. Unraveling heme detoxification in the malaria parasite by in situ correlative X-ray fluorescence microscopy and soft X-ray tomography. Sci. Rep. 7, 7610 (2017)

3 Pagola, S. et al. The structure of malaria pigment $\beta$-haematin. Nature 404, 307 (2000)

4 Straas $\varnothing$, T. et al. The Malaria Pigment Hemozoin Comprises at Most Four Different Isomer Units in Two Crystalline Models: Chiral as Based on a Biochemical Hypothesis or Centrosymmetric Made of Enantiomorphous Sectors. Cryst. Growth. Des. 14, 1543 (2014)

5 Straas $\varnothing$, T. et al. The Role of the Four Stereoisomers of the Heme Fe-O Cyclic Dimer in the Crystalline Phase Behavior of Synthetic Hemozoin: Relevance to Native Hemozoin Crystallization. Cryst. Growth. Des. 11, 3342 (2011)

6 Marom, N. et al. Structure and Formation of Synthetic Hemozoin: Insights From First-Principles Calculations. Cryst. Growth. Des. 11, 3332 (2011)

7 Kapishnikov, S. et al. Digestive Vacuole Membrane in Plasmodium falciparum-Infected Erythrocytes: Relevance to Templated Nucleation of Hemozoin. Langmuir 29, 14595 (2013)

8 Kapishnikov, S. et al. Oriented nucleation of hemozoin at the digestive vacuole membrane in Plasmodium falciparum. PNAS 109, 11188 (2012)

9 Kapishnikov, S. et al. Aligned hemozoin crystals on a curved surface in malarial red blood cells revealed by nanoprobe X-ray Fe-fluorescence and diffraction. PNAS 109, 11184 (2012)

10 Buller, R. et al. Quinoline Binding Site on Malaria Pigment Crystal: A Rational Pathway for Antimalarial Drug Design. Cryst Growth Des 2, 553 (2002)

11 Dubar, F. et al. The Antimalarial Ferroquine: Role of the Metal and Intramolecular Hydrogen Bond in Activity and Resistance. ACS Chem. Biol. 6, 275 (2011)

12 Kapishnikov, S. et al. Mode of action of quinoline antimalarial drugs in red blood cells infected by Plasmodium falciparum revealed in vivo. PNAS 116, 22946 (2019) 